\title{
The DDI Matures: 1997 to the Present
}

by Mary Vardigan ${ }^{1}$

\begin{abstract}
The Data Documentation Initiative (DDI) began in 1995 with a small international group coming together with a focus on social science metadata. The group moved quickly to develop a first specification, around which a community of practice emerged. That community, along with the DDI specification itself, has evolved over the last two decades to reflect developments in the social sciences, technological advances, and innovation in research practice. This paper chronicles the history of the DDI from its instantiation in XML in 1997 to its current status as the de facto standard for documenting data in the social and behavioral sciences.
\end{abstract}

Keywords Documentation, metadata, standards

\section{Note of Acknowledgment}

The centrality of good documentation to effective social science has been a key tenet of the IASSIST vision throughout its history. Since hosting the inaugural meeting of what was to become the Data Documentation Initiative in Quebec City in 1995, IASSIST has nurtured and promoted the DDI effort every step of the way. Indeed, the DDI story cannot be told properly without acknowledging the support of the IASSIST community through the years. The pioneering efforts and leadership of Sue Dodd deserve special recognition. Her work in data citation and structured metadata inspired a community and set the stage for developments like DDI. Fittingly, in 1993 Sue received the first ICPSR-sponsored Warren Miller Award for Meritorious Service to the Social Sciences, which recognizes contributions to essential infrastructure. We continue to benefit from her foundational work and wisdom.

\section{Introduction}

When our story left off (see Ann Green and Chuck Humphrey's article in this issue), the Data Documentation Initiative (DDI) specification had just been translated from SGML to XML in 1997, and it was moving toward official publication. There was a strong sense in the community that DDI XML, given its rich and structured nature, could be used to drive process, and that its coverage could and should be extended to document more complex datasets.

What happened next? How did the DDI Committee go on to enhance and augment the specification to meet rising expectations? How did the community of practice grow to encompass users in over 70 countries around the world? And how did infrastructure surrounding the DDI, including sustainable support for the organization itself and tools to make use of DDI, come into being? This paper describes the ways in which the DDI initiative addressed these challenges from 1997 to the present, ending with a view into what the future holds for DDI as we move forward.

\section{Publishing DDI Version 1}

As we have seen, DDI began as a volunteer effort, drawing on metadata expertise and interest from across the social science research community. In-kind contributions made it possible for DDI to be instantiated as a specification with a user community actively working around it.

However, in-kind contributions cannot provide the type of sustainable structure needed to fund face-toface meetings and development work, and thus the DDI Committee and its founders decided to pursue external funding streams. In 1997 the Inter-university Consortium for Political and Social Research (ICPSR) applied to the National Science Foundation (NSF) under 
its "Infrastructure in the Social Sciences" program and received an award that included funds for enhancing and testing the DDI specification. Having convened the first DDI Committee two years earlier, ICPSR was the "home" for DDI, providing administrative and substantive support.

The beta-test of the DDI DTD began in March 1999 and continued until August. Betatesters ${ }^{2}$ received financial support to test the specification and to report on their findings.

At the conclusion of the beta-test, a list of changes suggested by the testers was compiled and subsequently reviewed at a meeting of the DDI Committee held in October 1999. Version 1 of the DTD, incorporating these changes, was published March 24, 2000.

\section{Extending Version 1}

With Version 1 published and external support for DDI activities ending, the DDI Committee needed to find new funding sources. While an independent review funded by NSF found that DDI was a "worthwhile scientific effort that filled an urgent need for standardization of social science technical documentation and interoperability" (indeed, one evaluator termed it "a strategic component of the infrastructure necessary to support the exchange of structured social research survey data" [ICPSR 2001]), it was difficult to obtain funding for this type of endeavor. Health Canada came to the rescue, providing a substantial amount of financial support during 2001-2002 to enable the DDI Committee to meet and to make improvements to the specification, most notably additions related to aggregate data and geography.

Aggregate data was the focus of a small Working Group meeting in April 2001 in Voorburg, the Netherlands. Agreement was reached during that meeting on a draft aggregate data model (also known as "ncubes"), which was reviewed by the Committee at a meeting in Washington, DC, held in June 2001. Committee members began testing the new Version 1.02 of the DTD, with the extension describing aggregate/tabular data. Several other changes were made to study- and variable-level elements. By March 2003, Version 2 of the specification was published, with these enhancements:

- Aggregate extension elements (nCubes and location map)

- Internal formatting elements from the TEl specification to permit formatting within elements

- New geographic elements: Geographic Bounding Polygon,

Polygon, Point, G-Ring Latitude, G-Ring Longitude, and

Geographic Map

\section{The DDI Alliance Emerges}

It was becoming clear that generating external funding to support continued development of the DDI specification would be challenging so another approach was considered: becoming a self-sustaining membership Alliance, modeled along the lines of the successful World Wide Web Consortium.

In June 2002, the DDI Committee met in Storrs, CT, in conjunction with the IASSIST meeting. The main focus of this meeting was a draft charter, written by Richard Rockwell, to create a DDI Alliance - a new membership structure and funding base that would provide support so that the initiative could continue. The charter document provided for an Expert Committee with representation from the DDI Alliance membership, with each member of the Committee having a vote and thus a say in the future of the DDI. A
Steering Committee to provide oversight was also established via the charter.

The final meeting of the original DDI Committee ${ }^{3}$ was held in February 2003, in Washington, where the group approved numerous changes to the DTD leading to the publication of DDI 2.0 (see above). An Open Meeting of the DDI Alliance was held in conjunction with IASSIST in Ottawa in May 2003. Meeting participants discussed the new Alliance structure and elements of a Strategic Plan for the next three years of the Alliance.

\section{Toward a Lifecycle Specification}

Meanwhile, expectations around what the DDI could document were growing. A page on the DDI Web site ("About the Specification") in 2003 provides this vision for the DDI:

"The DDI aims to be the foundation for collection, distribution, use, and archiving of many future data collection projects in the social and behavioral sciences, across institutions, countries, and disciplines. It also aims to be the basis for retrofitting documentation of older studies for improved ease of use and stronger guarantee of archival preservation."

At its first meeting in 2003, the new DDI Expert Committee picked up this vision and set an agenda for the future that was ambitious and comprehensive.

First, the committee discussed the need for a data model. It was generally agreed that the XML Document Type Definition (DTD) for the DDI had limitations: it was not as modular and easily extensible as it should be and it had not been thoroughly reviewed for internal logic. Having a model, most likely in Universal Markup Language (UML), to reflect the underlying design and structure of the specification would represent a big step forward. With such a model, the DDI could be expressed as XML Schema, RDF, a DTD, or possibly other formats. The Health Canada/Nesstar partnership had already done some work on a data model for its DAIS/nesstar software that harmonized the DDI with ISO 11179.

Employing XML Schemas to express DDI was also discussed. Moving the DTD to a Schema to take advantage of the modularity in Schemas, the capability for local extension, and the flexibility of namespaces was considered essential to the DDI's continuing evolution. ICPSR and Harvard-MIT Data Center had been working on a Schema version of the DDI that incorporated all of the documentation found in the Tag Library as well as the DTD comments.

It was pointed out that the Alliance could not abandon the DTD since a lot of markup had been done that was compliant with Versions 1 and 2. The Alliance discussed the need to proceed on parallel tracks, moving the DTD along from Version 2.0 to subsequent iterations in that development line at the same time that a modular Version 3 was developed.

The group also reviewed the Statistical Data and Metadata eXchange (SDMX), a project to develop an interchange format for time series data and metadata. The SDMX initiative was viewed as a natural partnership, and it would come to be seen as complementary to DDI (Gregory and Heus, 2007). Aligning with the MetaDater and MADIERA projects in Europe was another topic raised. 
To carry forward this ambitious program of work, new working groups were formed:

- Structural Reform Working Group (later called the Technical Implementation Committee [TIC] and now the Technical Committee $[\mathrm{TC}])$, which would take on the task of "schematizing" DDI

- Substantive Content Working Group, broken out into:

- Group 1: Aggregate Data, Geography \& Time

- Group 2: Comparative Data/Families of Datasets

- Group 3: Complex Files

- Group 4: Instrument Documentation

- Usability and Outreach Working Group

This ambitious agenda set the stage for DDI 3, which was five years in the making. At its meeting in 2004 in Madison, Wisconsin, the Expert Committee discussed the coverage and scope for DDI 3 and introduced the concept of a lifecycle model. This model (see Figure 1) was innovative for its time; subsequently the notion of the data lifecycle became an integral part of the discourse around research data management. The DDI Lifecycle approach would come to influence the Generic Statistical Business Process Model (GSBPM) used by national statistical institutes as a framework for data production (Vale, 2010).

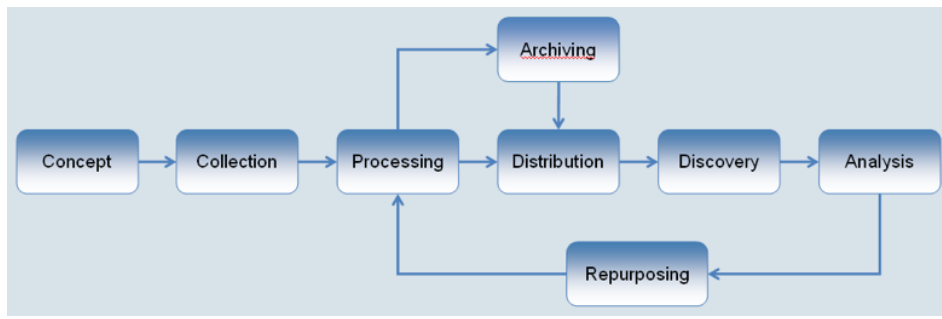

Figure 1: DDI Lifecycle Model

Not everyone was on board with a move to this lifecycle model. A considerable investment had been made in DDI 2.X and people were understandably reluctant to support a new specification that would in effect shift attention and resources away from this version However, in 2005, at the meeting in Edinburgh, the Alliance ratified the lifecycle model and DDI 3 began to take shape.

A public review of DDI 3 took place in 2007 and the specification was published in 2008 as XML Schemas. It was a radical departure from DDI 2.X in many ways. First, it was designed to be used by developers with machine-actionability in mind. While DDI 2.X could be understood and implemented by data librarians, DDI 3 was more complex, often requiring a higher level of technical expertise.

The specification itself was designed to be modular and to document and manage different stages of the data lifecycle. It was predicated on the principle of reusing metadata to eliminate costly redundancies and support explicit comparison (Vardigan, Heus, and Thomas, 2008). As Green and Humphrey note, "enter once and use many times" is a powerful paradigm, which DDI 3 exploited through referencing. As an example, response categories can be defined once and then used multiple times by both questions and variables.

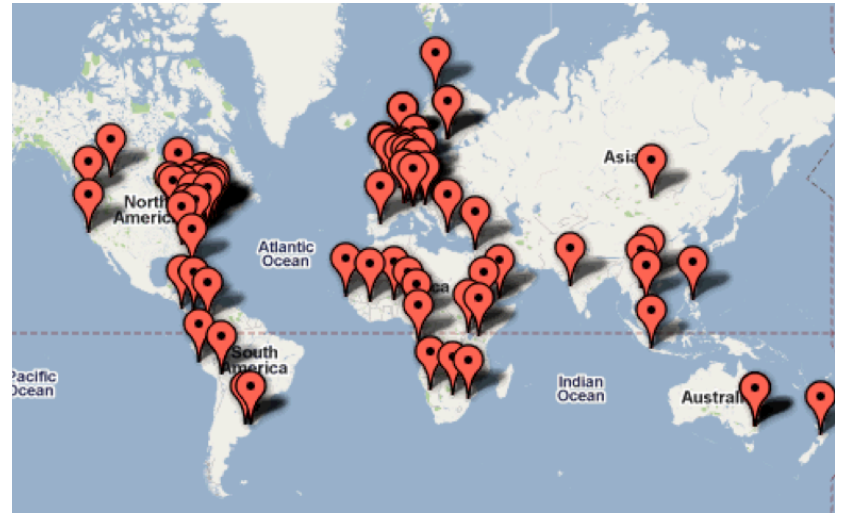

Figure 2: Organizations using DDI

DDI 3 also aligned with several other metadata standards including ISO 11179, SDMX, geographic and spatial standards, Dublin Core, and others

\section{The Community Expands}

As the DDI community of practice began to integrate DDI 3 into its work after 2008, interest from new audiences, including national statistical institutes (NSIs), other data producers, and developers and implementers, became evident and new DDI projects sprang up, many of which were discussed in IASSIST presentations, workshops, and posters. Uptake of DDI 2.X continued as well, resulting in DDI spreading across the globe. As a result of the World Bank-supported International Household Survey Network (IHSN) program and its incorporation of DDI into documentation tools, DDI came to be used in over 70 countries, many in the developing world (see Figure 2).

\section{Bringing users together}

DDI users were eager to meet in a forum where ideas, innovations, and knowledge of DDI could be shared. Led by Joachim Wackerow of GESIS-Leibniz Institute for the Social Sciences and Nikos Askitas of the Institute for the Study of Labor (IZA) in Germany, the first European DDI User Meeting (EDDI), cosponsored by GESIS and the IZA, took place in Bonn, Germany, in December 2009. Subsequent meetings took place in Utrecht, Netherlands; Gothenburg, Sweden; Bergen, Norway; and Paris, France, with the 2014 EDDI slated to take place in London.

In 2013 the concept of a DDI user meeting spread to North America, with the first NADDI conference taking place at the University of Kansas, organized by Larry Hoyle with funding from the Alfred P. Sloan Foundation. NADDI 2014 will be held in Vancouver.

\section{Teaching about DDI}

DDI training, which had been taking place around IASSIST and in other venues since 2001, was expanded in response to DDI 3, with Joachim Wackerow of GESIS organizing annual training and workshops at Schloss Dagstuhl, Leibniz Center for Informatics, an IT retreat center in Wadern, Germany, during the last quarter of each year. The first such training took place in 2007. Typically, training in DDI 3 is held for a week, followed by a workshop on a dedicated topic.

\section{Developing DDI tools}

Tools are key to a metadata standard's success: if markup cannot be produced efficiently, a standard may not find an audience. DDI owes much of its success to the parallel development of the DDI 
markup and data analysis tool Nesstar, created by the Norwegian Social Science Data Service (NSD) for use with DDI Codebook (NSD, 1999). Nesstar Publisher formed the basis for a toolkit designed to assist data producers in developing countries in documenting and disseminating data (see the IHSN discussion above). Dataverse Network, developed by Harvard-MIT, also adopted DDI, both as a standard for the study-level metadata entered at deposit and as a foundation for variable-level analysis. Support for DDI 2 was also incorporated into the Survey Documentation and Analysis (SDA) online analysis system created at the University of California, Berkeley.

Other innovative tools were developed with a focus on DDI Lifecycle. For example, the Michigan Questionnaire Documentation System (MQDS) exports DDI Lifecycle from Blaise Computer-Assisted Interviewing software. StatTransfer, a commercial product that transfers data among software packages, now also exports DDI 3.

The Danish Data Archive developed a DDI editor and Colectica developed a suite of tools that includes software to produce, view, and edit DDI Lifecycle metadata with an interface to CAl tools. Colectica for Excel was also added, permitting researchers working in Excel to create study descriptions and document their data at the variable level.

Database tools for DDI like Questasy, developed at CentERdata in the Netherlands, added new options for DDI users. The recently released Sledgehammer tools suite developed by Metadata Technology facilitates the transformation of data across formats and enables the extraction and generation of DDI metadata. Developers of many of these DDI-enabled tools have begun to meet periodically during the year at conferences to share ideas and to keep each other informed as new tools are created.

\section{Stepping Back: An Evaluation Takes Place}

The flurry of activity after DDI Lifecycle was released and the increasing diversity and expectations of new audiences led the DDI Alliance to initiate an open and independent review of the DDI initiative to inform its evolution going forward. There was a sense that the Alliance had matured since its inception in 2003 and that the organization needed to restructure to align with its accomplishments in order to be equipped to address new challenges.

Thus, in 2010, at the request of the DDI Alliance members, the DDI Steering Committee initiated a thorough and independent review of DDI governance and IP issues. The Steering Committee contracted with Breckenhill Inc. to provide a review under the following terms of reference:

- Clarify the intellectual property rights to the DDI specification and how the Alliance can best protect its IP

- Consider alternatives to the current Alliance governance structure

- Review the structure of host institutions and associations described in the Bylaws with a view toward opening up the Alliance to others to participate in governance

- Review the Bylaws and rewrite to be more specific on the above points

- Provide guidance on having a Constitution that does not change and Bylaws that are easier to change, separating the mechanism for revising the specification from the Bylaws
- Review the membership agreement and suggest content

- Suggest content of a contributor agreement for those contributing products to the Alliance

- Review the current Conflict of Interest form used by the Alliance and provide guidance on how the Alliance should approach this broad area

After interviewing a large group of DDI stakeholders and consulting widely on legal issues, Breckenhill provided a report to the Steering Committee in May 2011 detailing the findings related to the above questions (Breckenhill Inc., 2011).

In response to the review, the Alliance drafted a new Charter and Bylaws, which went into effect in July 2013. These new Bylaws outlined an organization that is broadly representative of the membership and structured to support the effective development of the DDI specifications. There is an Executive Board elected by the member representatives, a Scientific Board that oversees the substantive development of the DDI specifications, and a Technical Committee that creates and stewards the specifications and ensures their usability.

The revised Bylaws also allow for the DDI Alliance to be instantiated within the University of Michigan as an organizational host. This arrangement permits the U-M to protect the intellectual property of the Alliance and provides a home for the DDI Alliance Secretariat through the Inter-university Consortium for Political and Social Research (ICPSR).

DDI also rebranded its specifications after the review to give an indication of their scope: DDI 1.X and 2.X became DDI Codebook, while DDI 3.X became DDI Lifecycle.

\section{DDI Moving Forward: What Lies Ahead}

\section{Attracting new audiences}

With the publication of DDI Lifecycle, there was a surge of interest in DDI by many of the national statistical institutes and organizations around the world, and the DDI Alliance now counts the U.S. Bureau of Labor Statistics, Statistics New Zealand, the Australian Bureau of Statistics, the French National Institute of Statistics and Economic Studies, the Food and Agriculture Organization of the United Nations, and Eurostat as DDI members.

The DDI Alliance began working with NSIs in some notable ways with important synergies emerging. The SDMX-DDI Dialogue project helped to surface the similarities and differences between the two standards (many NSIs mandate the use of SDMX), and the DDI Alliance formally endorsed a collaboration with the SDMX community to enable the two standards to work together.

The Alliance has also supported development of the Generic Statistical Information Model (GSIM), the first internationally endorsed reference framework for statistical information that NSIs are using to inform the modernization of the production of official statistics. The Alliance has made an offer of support to work together on an implementation model for GSIM.

\section{Integrating with the semantic web}

Work is under way on two RDF (Resource Description Framework) vocabularies, the DDI-RDF Discovery vocabulary for publishing metadata about datasets into the Web of Linked Data, and XKOS, an RDF vocabulary for describing statistical classifications, which is an extension of the popular SKOS (Simple Knowledge Organization 
System) vocabulary. The public review of both vocabularies is planned for 2014

\section{Developing the next-generation DDI}

While the existing DDI Codebook and Lifecycle specifications continue to be fine-tuned (DDI Codebook Version 2.5.1 [in Schema form] and DDI Lifecycle 3.2 were published in early 2014), the Alliance has begun another ambitious project - to create a DDI specification based on an information model. The Alliance supports this move to a model-based specification as it will provide greater flexibility: the model can be expressed in a variety of technical formats including XML Schema, RDF/OWL Ontology, relational database schema, and other languages. Also, having a model will make it easier to understand the specification, to interact with other disciplines and other standards, to develop and maintain it in a consistent and structured way, and to enable software development that is less dependent on specific DDI versions. Interestingly, creating a data model was a component of the original agenda for development of DDI 3, so the initiative has come full circle.

The Alliance has other goals for this new model-based DDI: this is an opportunity to respond to community expectations by creating a new version of the specification that can transcend traditional disciplinary barriers to document data about humans and their impact more broadly. As an example, while data collection instruments in the social sciences have traditionally been surveys, we can also view blood pressure gauges and magnetic resonance imaging (MRI) scans as new types of instruments that capture and export data. There is also a growing emphasis on documenting data from administrative registers and various Internet sources. In addition, the next-generation DDI will ultimately add coverage in several new areas:

- Abstraction of data capture/collection/source with "plug-ins" to handle different types of data

- New content on sampling, survey implementation, weighting, and paradata

- New content pertaining to qualitative data

- Framework for data and metadata quality

- Framework for access to data and metadata

- Process (work flow) description across the data life cycle, including support for automation and replication

- ntegration with existing standards like GSBPM/GSIM, SDMX, CDISC, Triple-S

- Disclosure review and remediation

- Data management planning

Work on the model began in October 2013 when a group convened at Schloss Dagstuhl to focus on gathering requirements for and modeling this next-generation DDI. As part of a paper summarizing the requirements (DDI Working Paper Series No. 4), the group articulated a set of design principles for the information model that reflect what the Alliance has learned over the years about effective standards and their development:

1. Simplicity - The model is as simple as possible and easily understandable by different stakeholders.

2. User Driven - User perspectives inform the model to ensure that it meets the needs of the international DDI user community.

3. Terminology - The model uses clear terminology and when possible, uses existing terms and definitions.
4. Iterative Development - The model is developed iteratively, bringing in a range of views from the user community.

5. Documentation - The model includes and is supplemented by robust and accessible documentation.

6. Lifecycle Orientation - The model supports the full research data lifecycle and the statistical production process, facilitating replication and the scientific method.

7. Reuse and Exchange - The model supports the reuse, exchange, and sharing of data and metadata within and among institutions.

8. Modularity - The model is modular and these modules can be used independently.

9. Stability - The model is stable and new versions are developed in a controlled manner.

10. Extensibility - The model has a common core and is extensible.

11. Tool Independence - The model is not dependent on any specific IT setting or tool.

12. Innovation - The model supports both current and new ways of documenting, producing, and using data and leverages modern technologies.

13. Actionable Metadata - The model provides actionable metadata that can be used to drive production and data collection processes.

\section{Conclusion}

From its modest start in Quebec City in 1995 with 23 individuals around the table, the DDI initiative has accomplished some important objectives, producing two development lines to document social science research data. The work continues, with a new, more ambitious goal: to spread the next-generation DDI across the social and behavioral sciences and into new communities to ensure the effective documentation of research data and its future use.

DDI will mark its 20-year anniversary in 2015. With almost two decades of experience, the DDI community has learned a lot about metadata standards development, and the lessons learned can inform what lies ahead. The journey is sure to be interesting and we welcome fellow metadata travelers, both within IASSIST and beyond.

\section{References}

"About the [DDI] Specification." DDI Alliance Web Site (via the Internet Archive Wayback Machine): <http://web.archive.org/ web/20031112160748/www.icpsr.umich.edu/DDI/codebook/index. html> (Accessed February 12, 2014)

About the MADIERA Project: <http://www.ist-world.org/ProjectDetails. aspx?Projectld=2bbe31 ebd47c4edfa7c4465b7e9ee8ca\&SourceData baseld=9cd97ac2e51045e39c2ad6b86dce1ac2> (Accessed February 12, 2014)

About the Metadater Project: <http://www.ist-world.org/ProjectDetails. aspx?Projectld=2bbe31ebd47c4edfa7c4465b7e9ee8ca\&SourceData baseld=9cd97ac2e51045e39c2ad6b86dce1 ac2 > (Accessed February 12, 2014)

Breckenhill, Inc. (2011) DDI Alliance External Review: Summary and Recommendations July 8, 2011. <http://www.ddialliance.org/sites/ default/files/DDI\%20Alliance\%20Review\%20Summary\%202011-0708.pdf>

DDI Alliance Original Charter (via the Internet Archive Wayback Machine): <http://web.archive.org/web/20040324061527/http:/ www.icpsr.umich.edu/DDl/org/charter.html> (Accessed February 12, 2014) 
DDI Tools Catalog: <http://www.ddialliance.org/resources/tools> (Accessed February 12, 2014)

Green, Ann, and Humphrey, Chuck (2013) "Building the DDI." IASSIST Quarterly. Vol 37.

Gregory, Arofan and Pascal Heus." (2007) DDI and SDMX:

Complementary, Not Competing, Standards." Open Data Foundation Paper, <http://odaf.org/papers/DDI_and_SDMX.pdf>

Inter-university Consortium for Political and Social Research (ICPSR). (2001) Addendum to NSF Final Report "Electronic Preservation of Data Documentation: Complementary SGML and Image Capture," SBR-9617813: Results of the Evaluation of the Data Documentation Initiative (DDI), April 24, 2001. <http://www.ddialliance.org/sites/ default/files/evalsummary.pdf>

Minutes of First Meeting of DDI Alliance Expert Committee, October 12-13, 2003, Ann Arbor, Michigan <http://www.ddialliance.org/DDI/ committee-info/minutes/2003-10-12.html> (Accessed February 12, 2014)

Norwegian Social Science Data Services (NSD). (1999) “Providing Global Access to Distributed Data Through Metadata Standardisation - The Parallel Stories of Nesstar and the DDI" (Working Paper \#10). UN/ECE Work Session on Statistical Metadata (Geneva, Switzerland, 22-24 September 1999).

$<$ http://www.google.com/url?sa=t\&rct=j\&q=\&esrc=s\&source=we $b \& c d=1 \& c a d=r j a \& v e d=0 C C s Q F j A A \& u r l=h t t p \% 3 A \% 2 F \% 2 F w w w$. unece.org\%2Fstats\%2Fdocuments\%2F1999\%2F09\%2Fmetis\%2 F10.e.pdf\&ei=tSCvUuLyD6GfyQGgs4CABA\&usg=AFQjCNEKWB xQdJRqbJrRPZadj5JDBPIRMw\&sig2=Q8GsTkpWSH5B2XQzCmFLdA\&bvm $=$ bv. 57967247, d.aWc $>$

Participants in 2012 Dagstuhl Seminar on DDI Moving Forward. (2012) "Developing a Model-Driven DDI Specification." DDI Working Paper Series, Paper No. 4.

<http://dx.doi.org/10.3886/DDIWorkingPaper04>

Vale, Steven, United Nations Economic Commission for Europe. (2010) "Exploring the Relationship Between DDI, SDMX and the Generic Statistical Business Process Model." < http://dx.doi.org/10.3886/ DDIOtherTopics01>

Vardigan, Mary, Pascal Heus, and Wendy Thomas. (2008) “Data Documentation Initiative: Toward a Standard for the Social Sciences." International Journal of Digital Curation. Vol. 3, No. 1, pp. 107-113 <http://dx.doi.org/10.2218/ijdc.v3i1.45>

\section{Notes}

1, Mary Vardigan is an Assistant Director at the Inter-university Consortium for Political and Social Research (ICPSR) and Director of the DDI Alliance. She can be reached at vardigan@umich.edu.

2. Beta-esters of the first DDI specification included the following institutions:

- Centre for Comparative European Survey Data (CCESD) Contact: Richard Topf

- Danish Data Archive - Contact: Nanna Floor Clausen

- The (UK) Data Archive - Contact: Ken Miller

- Harvard-MIT Data Center - Contacts: Michael McDonald, Micah Altman

- NIWI-Steinmetz Archive - Contact: Repke de Vries

- Norwegian Social Science Data Services (NSD) - Contact: Jostein Ryssevik

- University of California, Berkeley, Survey Research Center Contact: Juteh Theresa Cheng, Jeff Royal

- University of Giessen - Contact: Karsten D. Wolf

- University of Ljubljana, Social Science Data Archive - Contact: Janez Stebe

- University of Michigan, Harlan Hatcher Library - Contacts: Bonnie Dede, JoAnn Dionne, Lynn Marko, Patricia Dragon
- University of Minnesota, Machine Readable Data Center Contact: Wendy Treadwell

- University of Warsaw, Institute for Social Studies - Contacts: Pawel Morawski and Jacek Szamrej

- University of Wisconsin-Madison, Data and Program Library Service - Contact: Cindy Severt

3. Members of the DDI Committee when it met for the last time in February 2003 included: Bjorn Henrichsen, Chair, Norwegian Social Science Data Services; Micah Altman, Harvard University; Atle Alvheim, Norwegian Social Science Data Services; Grant Blank, American University; Ernie Boyko, Statistics Canada; Bill Bradley, Health Canada; Cavan Capps, Bureau of the Census; Bill Connett, University of Michigan; Cathryn Dippo, Bureau of Labor Statistics; Pat Doyle, Bureau of the Census; Dan Gillman, Bureau of Labor Statistics; Peter Granda, ICPSR; Ann Green, Yale University; Peter Joftis, ICPSR; Ken Miller, ESRC Data Archive; Tom Piazza, University of California, Berkeley; Karsten Boye Rasmussen, University of Southern Denmark; Richard Rockwell, The Roper Center; Jostein Ryssevik, Norwegian Social Science Data Services; Merrill Shanks, University of California, Berkeley; Peter Solenberger, University of Michigan; Wendy Thomas, University of Minnesota; Rolf Uher, Zentralarchiv für Empirische Sozialforschung; Mary Vardigan, ICPSR

\section{Appendix: Past Chairs, Vice Chairs, and DTD and Schema Authors}

\section{DDI Committee Chairs}

- Merrill Shanks, University of California, Berkeley: 1995-2002

- Bjorn Henrichsen, Norwegian Social Science Data Service (NSD): 2002-2003

\section{DDI Alliance Expert Committee Chairs and Vice Chairs}

- Tom Piazza, University of California, Berkeley: 2003-2005

- Hans Jorgen Marker, Danish Data Archive (DDA), Chair, and Ron Nakao, Stanford University, Vice Chair: 2005-2010

- Chuck Humphrey, University of Alberta, Chair, and Mari Kleemola, Finnish Social Science Data Service (FSD), Vice Chair: 2010-2013

\section{DDI Executive Board and Membership Chair and Vice \\ Chair}

- Gillian Nicoll, Australian Bureau of Statistics, Chair, and Ron Nakao, Stanford University, Vice Chair: 2013-

\section{DTD and Schema Authors (in order of contributions)}

- David Barber, University of Michigan

- John Brandt, University of Michigan

- Ann Green, Yale University

- Paul Schaffner, University of Michigan

- Nancy Vlahakis, University of Michigan

- Daniel Pitti, University of California, Berkeley

- Jan Nielsen, Danish Data Archive

- Jerome McDonough, University of California, Berkeley

- Perry Roland, University of Virginia

- Sanda lonescu, ICPSR

- Mark Diggory, Harvard-MIT Data Center

- Wendy Thomas, University of Minnesota

- Arofan Gregory, Metadata Technology 